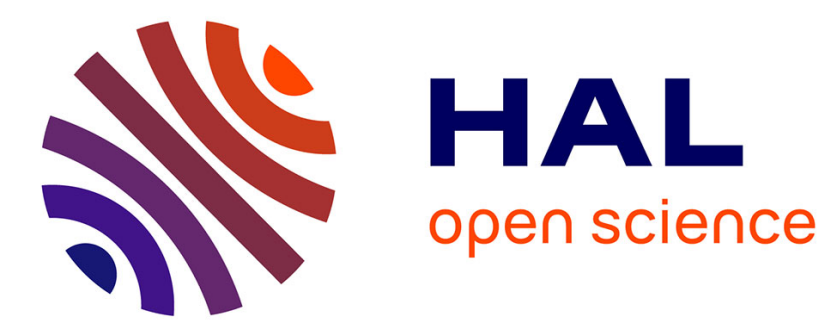

\title{
Successive Full-waveform inversion of surface seismic and seismic-while-drilling datasets without low frequencies,
}

N Kazemi, Jean Auriol, K Innanen, R Shor, I Gates

\section{To cite this version:}

N Kazemi, Jean Auriol, K Innanen, R Shor, I Gates. Successive Full-waveform inversion of surface seismic and seismic-while-drilling datasets without low frequencies,. 82 nd EAGE Conference \& Exhibition, 2020, Amsterdam, Netherlands. hal-02941015

\section{HAL Id: hal-02941015 https://hal.science/hal-02941015}

Submitted on 16 Sep 2020

HAL is a multi-disciplinary open access archive for the deposit and dissemination of scientific research documents, whether they are published or not. The documents may come from teaching and research institutions in France or abroad, or from public or private research centers.
L'archive ouverte pluridisciplinaire HAL, est destinée au dépôt et à la diffusion de documents scientifiques de niveau recherche, publiés ou non, émanant des établissements d'enseignement et de recherche français ou étrangers, des laboratoires publics ou privés. 


\title{
Th_Dome5_03
}

\section{Successive Full-Waveform Inversion of Surface Seismic and Seismic-While-Drilling Datasets without Low Frequencies}

\author{
N. Kazemi ${ }^{1 *}$, J. Auriol ${ }^{2}$, K. Innanen ${ }^{1}$, R. Shor ${ }^{1}$, I. Gates ${ }^{1}$ \\ ${ }^{1}$ University of Calgary; ${ }^{2}$ Centre National de la Recherche Scientifique
}

\section{Summary}

We have developed a full waveform inversion (FWI) algorithm for the seismic-while-drilling (SWD) dataset. Full waveform inversion is a local optimization method. To avoid the local minima, seismic data should have rich lowfrequency content. However, in the real-world, seismic data lacks low-frequency content, and FWI struggles to provide accurate subsurface properties. To remedy this shortcoming, we use SWD data to compensate for the lack of low frequencies in the surface seismic dataset. In SWD data, the drillbit acts as a seismic source. The drillbit generates significant elastic energy, which has different raypaths compared to the surface seismic. We show that if we understand the non-impulsive and correlative behavior of the drillbit source signature, the full waveform inversion of SWD data is possible. To estimate the drillbit source signature, we have developed a computational framework based on wave equation drill string dynamics modeling along with top-drive force and velocity measurements. Then, we feed the estimated dillbit source signature to the FWI algorithm to invert the SWD dataset. Our results show that the successive inversion of surface seismic and SWD datasets can compensate for the lack of low frequencies in the surface seismic data and reduce the uncertainties of the subsurface properties. 


\section{Introduction}

Full waveform inversion (FWI) (Virieux and Operto, 2009) is a computational framework that transforms the recorded seismic data to the high-resolution rock properties of the subsurface. FWI is an ongoing and active area of research in industry and academia, and it plays a promising and increasingly relevant role in oil/gas exploration. FWI is a nonlinear and ill-posed optimization problem and requires specific criteria such as low-frequency seismic data, dense acquisition geometry, and efficient solvers. These criterions usually are not satisfied in real-world applications. To rectify this, the seismic-while-drilling (SWD) dataset, whose ray paths are different from the surface seismic, can be added to the surface seismic data to compensate for the incompleteness of conventional seismic acquisition. SWD dataset is complementary to surface data, and it brings an opportunity to address uncertainties of the conventional FWI approach by adding new measurements to the inversion problem. Because drilling is necessary for oil production, SWD data acquisition has low cost, and it does not interrupt the drilling process.

The drillbit- rock interaction generates significant elastic energy that propagates in the subsurface and can reach the surface or nearby wells. However, the drill bit source signature is different from the impulsive sources such as dynamite. In the past, researchers aimed at modeling and estimating the nonimpulsive and correlative signature of the drillbit-rock interactions (Poletto, 2005; Kazemi and Sacchi, 2014; Auriol et al., 2019). Provided that we understand the drillbit-rock interaction mechanism and can model/estimate the drill bit source signature, the full waveform inversion of SWD data is possible. Previously, we show that having access to SWD data, background velocity profile, and a model of drill bit source signature, enables us to provide the prestack depth images of such a dataset (Kazemi et al., 2018b). We also show that combining SWD data with surface seismic data improves the nonuniform illumination problem (Kazemi et al., 2018a). Here, we use the recently proposed approach based on drillstring dynamics modeling and top-drive measurements (velocity and force) to model/estimate the drill bit source signature (Auriol et al., 2019). Then, we show the possibility of regularized fullwaveform inversion of SWD data and improvements in the resolution of FWI result when the algorithm is successively applied on surface seismic and SWD datasets.

\section{Full waveform inversion of SWD dataset}

The seismic-while-drilling forward modeling problem can be written as (Marfurt, 1984)

$$
\mathbf{M}(\mathbf{x}) \frac{\partial^{2} \mathbf{u}(\mathbf{x}, t)}{\partial t^{2}}+\mathbf{S}(\mathbf{x}) \frac{\partial \mathbf{u}(\mathbf{x}, t)}{\partial t}+\mathbf{D} \mathbf{u}(\mathbf{x}, t)=\mathbf{F}(\mathbf{x}, t)
$$

where $\mathbf{M}$ is the mass matrix, $\mathbf{S}$ is the stiffness matrix, $\mathbf{D}$ is the damping matrix, $\mathbf{F}$ is the drill bit source signature, $\mathbf{x}$ is the spatial coordinates, $t$ is time, and $\mathbf{u}$ is the seismic wavefield. Equation(1) in the frequency domain is

$$
\left[-\omega^{2} \mathbf{M}(\mathbf{x})+j \omega \mathbf{S}(\mathbf{x})+\mathbf{D}(\mathbf{x})\right] \mathbf{u}(\mathbf{x}, \omega)=\mathbf{F}(\mathbf{x}, \omega)
$$

where $\omega$ is angular frequency, and $j=\sqrt{-1}$ is complex identity. Hence, the wave equation in the frequency domain, i.e., Equation (2), can be expressed as linear system of equations

$$
\mathbf{B}(\mathbf{x}, \omega) \mathbf{u}(\mathbf{x}, \omega)=\mathbf{F}(\mathbf{x}, \omega)
$$

where $\mathbf{B}(\mathbf{x}, \omega)=-\omega^{2} \mathbf{M}(\mathbf{x})+j \omega \mathbf{S}(\mathbf{x})+\mathbf{D}(\mathbf{x})$ is the impedance matrix. The properties of subsurface such as velocity and density are embedded in the impedance matrix. Hence, the relationship between the subsurface physical properties and the seismic wavefield is nonlinear. The forward modeling reads

$$
\mathbf{u}=\mathbf{L}(\mathbf{m})
$$

where $\mathbf{m}$ is the vector of subsurface properties, and $\mathbf{L}$ is the forward modeling operator. Full waveform inversion is a local optimization problem which aims to estimate the subsurface properties by matching the recorded wavefield at the surface $\mathbf{u}_{o b s}$ to the modeled data $\mathbf{u}_{c a l}=\mathbf{L}(\mathbf{m})$. The simplest similarity measurement cost function can be written as

$$
J=\left\|\mathbf{u}_{o b s}-\mathbf{L}(\mathbf{m})\right\|_{2}^{2}+\alpha\|\mathbf{m}\|_{2}^{2}
$$


where $\alpha$ is a regularization parameter. Minimizing the cost function $J$ with respect to the subsurface model parameters $\mathbf{m}$ is a non-linear optimization problem. In real world applications, we usually implement iterative algorithms to solve this non-linear optimization problem. Here, we use L-BFGS method to find the desired solution $\mathbf{m}$ iteratively. As clear, one of the inputs of FWI inversion is the drill bit source function, i.e., F. Next section provides a methodology for estimating such a source function.

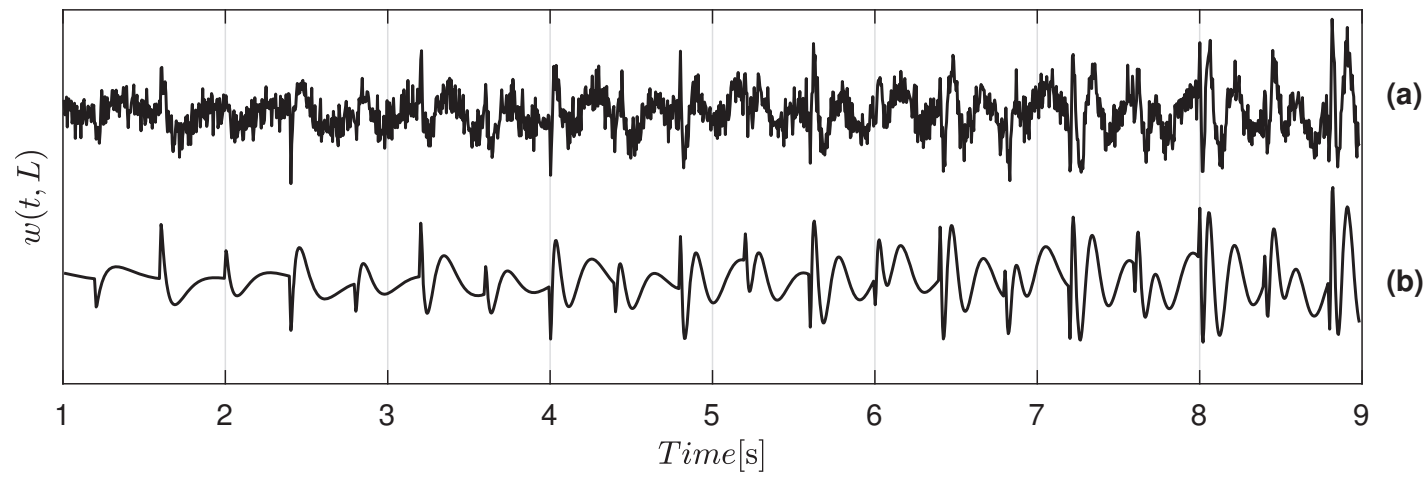

Figure 1: Drill bit source signatures. a) Simulated, and b) Estimated drill bit sources.

\section{Drill bit source signature modeling/estimation}

Recently Auriol et al. (2019) show that the drill bit source signature can be estimated through wave equation modeling of drillstring dynamics with topside and bit-rock boundary conditions combined with top-drive velocity and force measurements. Here, we use such a modeling engine to estimate the drill bit source signature necessary for SWD-FWI algorithm. To do so, we start with wave equation modeling of axial motion in the drillstring

$$
\frac{\partial^{2} \mathbf{u}(\mathbf{x}, t)}{\partial t^{2}}-c^{2} \frac{\partial^{2} \mathbf{u}(\mathbf{x}, t)}{\partial x^{2}}=-k \frac{\partial \mathbf{u}(\mathbf{x}, t)}{\partial t}
$$

where $c=\sqrt{\frac{E}{\rho}}$ is the velocity of drill pipe, $E$ is the Young's module, $\rho$ is drill pipe density, $k$ is the damping factor, and $\mathbf{u}(\mathbf{x}, t)$ is the axial motion in the drillstring in time $t$ and distance along drillstring $\mathbf{x}$. The axial force follows $\mathbf{w}(t, \mathbf{x})=A E \frac{\partial \mathbf{u}(\mathbf{x}, t)}{\partial x}$, and the axial velocity is $\mathbf{v}(t, \mathbf{x})=\frac{\partial \mathbf{u}(\mathbf{x}, t)}{\partial t}$. Then, the top-drive boundary condition can be written as

$$
-E A \frac{\partial \mathbf{u}(\mathbf{x}, t)}{\partial t}=\mathbf{w}(t, 0)
$$

where $\mathbf{w}(t, 0)$ is the topside weight function, and $A$ is the cross-sectional area of the drill pipe. Moreover, the drillbit-rock interaction follow

$$
M_{b} \frac{\partial \mathbf{v}(t, L)}{\partial t}=-\mathbf{w}_{b}(\mathbf{v}(t, L), \mathbf{w}(t, L))+\mathbf{w}(t, L)
$$

where $M_{b}$ is the mass of bottom hole assembly, $\mathbf{w}_{b}$ is the weight on bit, and $L$ is the length of drillstring measured from the surface to the drill bit. Through backstepping approach and by using top-drive measurements, the equations (6)-(8) can be solved for the drill bit source signature

$$
\begin{aligned}
w(t, L)= & \frac{E A}{2 c}\left(\mathrm{e}^{-\frac{k L}{2 c}} v\left(t-\frac{L}{c}, 0\right)-\mathrm{e}^{\frac{k L}{2 c}} v\left(t+\frac{L}{c}, 0\right)\right)+\frac{1}{2}\left(\mathrm{e}^{-\frac{k L}{2 c}} w\left(t-\frac{L}{c}, 0\right)+\mathrm{e}^{\frac{k L}{2 c}} w\left(t+\frac{L}{c}, 0\right)\right) \\
& +\int_{-\frac{L}{c}}^{\frac{L}{c}}\left(f_{u}(s)+f_{z}(s)\right) v(t-s, 0) d s+\int_{-\frac{L}{c}}^{\frac{L}{c}} \frac{c}{E A}\left(f_{u}(s)-f_{z}(s)\right) w(t-s, 0) d s
\end{aligned}
$$

where $f_{z}$ and $f_{u}$ are some functions that depend on drilling parameters. For more information, readers can refer to Auriol et al. (2019). Note that Equation (9) is valid for the axial motion of the drillstring and the rotational motion is ignored. To fully model the elastic drill bit source signature, we need to consider 
the rotational motion in the drillstring, deviations in the well geometry, and friction between the drill pipe and the well. Another alternative for estimating the drill bit source signature is to implement blind deconvolution algorithms such as the SMBD algorithm (Kazemi and Sacchi, 2014) that showed promise when applied to SWD data (Kazemi et al., 2018a).
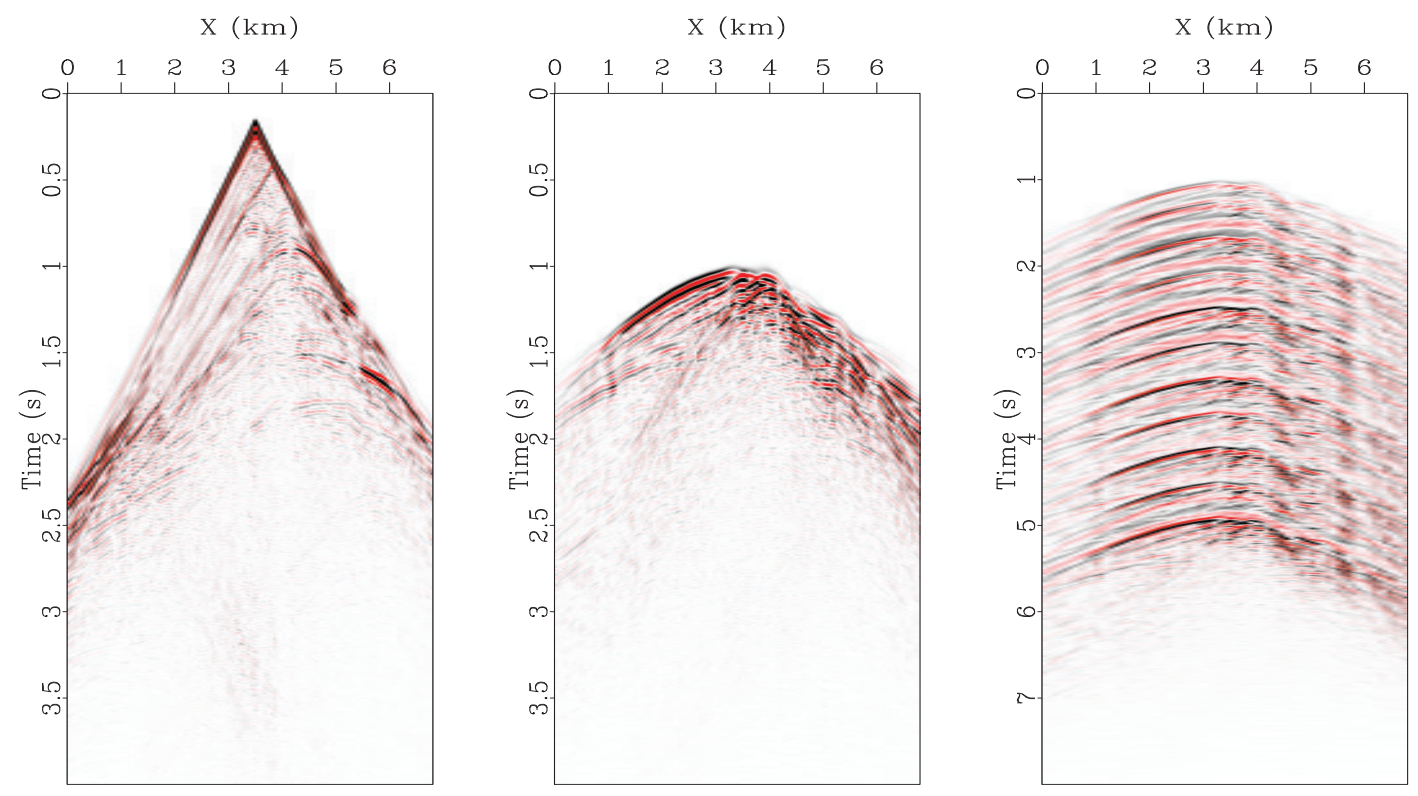

Figure 2: Surface and SWD shot gathers. Left) Surface seismic, Middle) Impulsive SWD, and Right) SWD shots.

\section{Examples}

To evaluate the performances of the full waveform inversion algorithm, we used the modified Marmousi2 model (Figure 3 upper-left). The model is $\approx 2.5 \mathrm{~km}$ in the depth direction and $\approx 7 \mathrm{~km}$ in the horizontal direction. The depth sampling and horizontal sampling are 10 meters. We deployed 66 surface seismic sources near the surface with a shot spacing of 100 meters. Acoustic finite difference modeling is used to generate synthetic seismic recordings. The generated shot gathers do not have free surface multiples (Figure 2 left). The receivers are active for all shots and are densely deployed near the surface with a receiver interval of $10 \mathrm{~m}$. A Ricker wavelet with the dominant frequency of $30 \mathrm{~Hz}$ is used as a source signature. Later, we implemented our frequency domain constant density acoustic full-waveform inversion algorithm to invert for the subsurface velocity. To make the inversion realistic and challenging, we assumed that the data lacks low-frequency components. Hence, we inverted 46 frequency realizations from $5-50 \mathrm{~Hz}$ with 500 iterations. Figure 3 upper-right shows the initial velocity. Due to the lack of low frequencies in the inversion process, the algorithm struggles to provide the accurate structure of the velocity profile in the deeper section of the model. Note that we are only inverting the reflection data.

To improve the performance of the FWI algorithm, we added SWD data to the inversion process. In the SWD data, we use the drill bit in the deeper part of the velocity model. We deployed 25 drill bit sources at the depth of $2.1 \mathrm{~km}$. The sources span from $2.4-3.6 \mathrm{~km}$ with the source spacing of $50 \mathrm{~m}$. We used a classical finite volume method based on a Godunov scheme to simulate the drill bit source signature (LeVeque et al., 2002). We considered noisy top-drive measurements with $S N R=10$. Then, Equation (9) is used to estimate the drill bit source signature. To simulate the SWD dataset, we used an acoustic finite-difference modeling engine and subsurface impulsive sources. Later, we convolved the impulsive SWD dataset (Figure 2middle) with the simulated drill bit source signature (Figure 2right). The estimated drill bit source signature is given as an input to our full-waveform inversion algorithm to invert the measured SWD data for the improved velocity model. In this case, we used the inverted velocity from the full waveform inversion of surface data as an initial solution. Again, 46 frequency realizations from $5-50 \mathrm{~Hz}$ are inverted with 500 iterations. Figure 3 lower-right shows the final result of inversion after successively using the surface-FWI and SWD-FWI algorithms. As clear, the velocity structure near the SWD sources in the deeper section of the model substantially improved. 

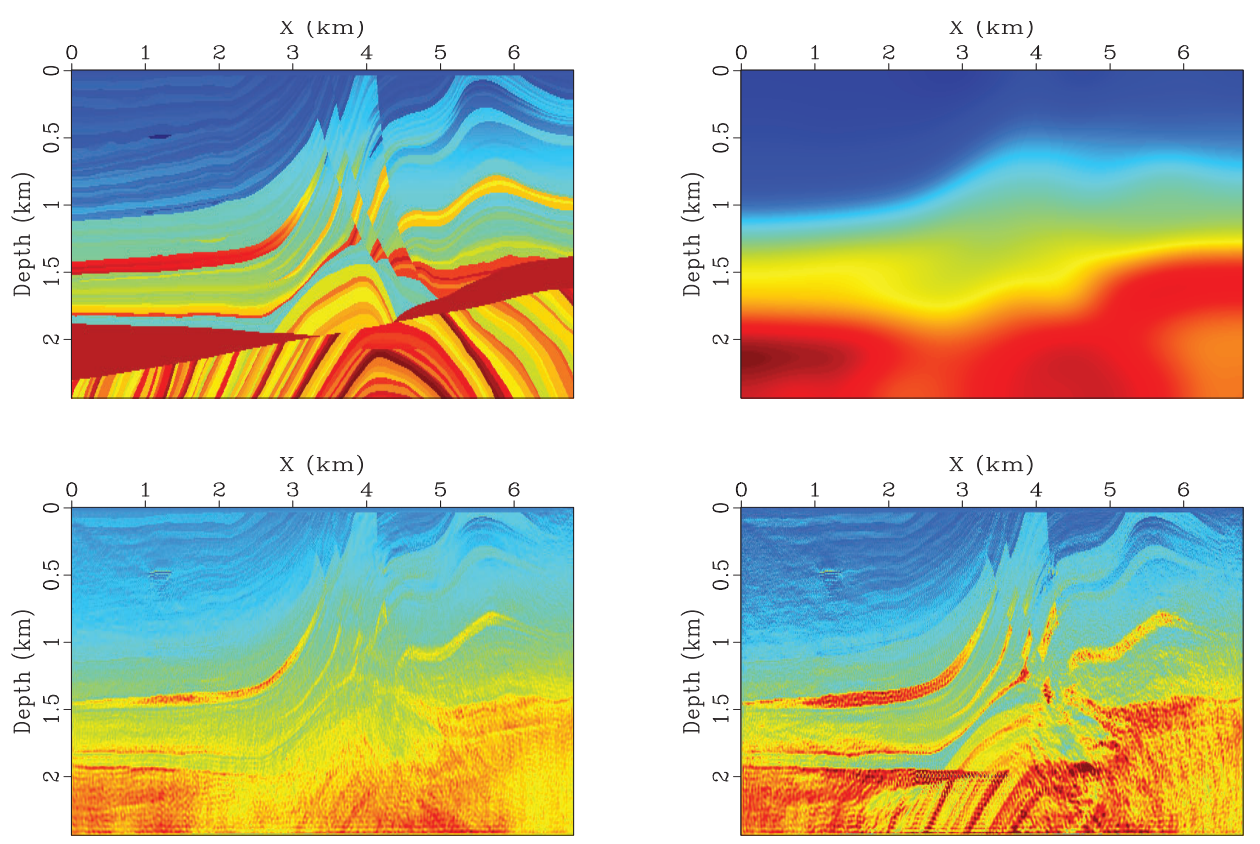

Figure 3: Performances of surface-FWI and SWD-FWI algorithms. Top-left) True model, Top-right) Initial model, Lower-left) surface-FWI, Lower-right) Successive surface-FWI and SWD-FWI.

\section{Conclusion}

We have developed a full waveform inversion algorithm for the SWD dataset. One of the main inputs of our algorithm is the source signature of the drill bit. Hence, we provided a modeling and estimation framework for the drill bit source signature. Our results showed that the lack of low frequencies degrades the performance of surface-FWI inversion and the algorithm could not resolve the deeper section of the model. To remedy this shortcoming, we successively inverted the surface and SWD datasets. The successive inversion method was able to recover the deeper section of the model.

\section{Acknowledgements}

This work was supported by the University of Calgary's Canada First Research Excellence Fund Program, the Global Research Initiative in Sustainable Low Carbon Unconventional Resources. The First author would like to thank Eldad Haber and Wenyong Pan for developing the conventional frequency domain FWI packages.

\section{References}

Auriol, J., Kazemi, N., Shor, R.J., Innanen, K.A. and Gates, I.D. [2019] A Sensing and Computational Framework for Estimating the Seismic Velocities of Rocks Interacting With the Drill Bit. IEEE Transactions on Geoscience and Remote Sensing, 1-12.

Kazemi, N. and Sacchi, M.D. [2014] Sparse multichannel blind deconvolution. Geophysics, 79(5), V143-V152.

Kazemi, N., Shor, R. and Innanen, K. [2018a] Illumination compensation with seismic-while-drilling plus surface seismic imaging. In: 80th EAGE Conference and Exhibition 2018. 1-5.

Kazemi, N., Shor, R. and Innanen, K. [2018b] Imaging with a seismic-while-drilling dataset. Proc. CSPG CSEG CWLS Conv, 1-4.

LeVeque, R.J. et al. [2002] Finite volume methods for hyperbolic problems, 31. Cambridge University Press.

Marfurt, K.J. [1984] Accuracy of finite-difference and finite-element modeling of the scalar and elastic wave equations. Geophysics, 49(5), 533-549.

Poletto, F. [2005] Energy balance of a drill-bit seismic source, part 1: Rotary energy and radiation properties. Geophysics, 70(2), T13-T28.

Virieux, J. and Operto, S. [2009] An overview of full-waveform inversion in exploration geophysics. Geophysics, 74(6), WCC1-WCC26. 